\title{
THE ASCA RESULTS OF GRO J1744-28
}

\author{
MAMIKO NISHIUCHI, YOSHITOMO MAEDA, KATSUJI KOYAMA AND \\ JUN YOKOGAWA \\ Department of Physics, Kyoto University, Sakyo, Kyoto, Japan \\ TADAYASU DOTANI, KAZUMI ASAI AND YOSHIHIRO UEDA \\ KAZUHISA MITSUDA, HAJIME INOUE AND FUMIAKI NAGASE \\ ISAS \\ AND \\ CHERISSA KOUVELIOTOU \\ Universities Space Research Association
}

\section{Introduction}

We present the ASCA/GIS results of the transient source GRO J174428 , the bursting X-ray pulsar, for the two times observations about one year apart. (the first and the second observations are on Feb.27th 1996 and Mar.16th 1997 respectively.) Since the discovery of GRO J1744-28 on Dec. 21995 with the BATSE observatory (Fishman et al. 1995; Kouveliotou et $a l$.), thousands of Type II X-ray bursts, and sinusoidal pulsations have been observed in the X-ray band. No other source has ever been found to exhibit such unusual characteristics. Following each burst the flux decreases below, and recovers to the pre-burst persistent level (here after the dip) within a few seconds to a few minutes, depending on the total flux of the burst. We examined the spectrum of GRO J1744-28 during each persistent, dip, burst phases at the two observations. This is the first detail study of the spectra (1-10keV band) of GRO J1744-28.

\section{Results and Discussion}

The persistent spectra of both observations are quite similar and the acceptable model for them is an absorbed power-law with a broad line whose center energy is around $6.7 \mathrm{keV}$ and with a soft black body component. Since the value of hydrogen column density is comparable to that of our 
Galactic center region, here and after we assume that GRO J1744-28 is near the Galactic center $(\mathrm{d}=8 \mathrm{kpc})$. At the first observation, GRO J1744-28 was as bright as the Eddington limit of a neutron star, but at the second observation, this source was about $\sim 1 / 5$ times bright as that of the first observation. The normalization of the black body component remain constant between these two observations. The size of this black body emission region is about the same as that of the surface of a neutron star. This suggests that the black body component is from the surface of the a neutron star. The gaussian center energy is constant from the first and second observations, but the gaussian sigma is broader at the first observation than at second one. The equivalent width of the Fe line at the first and second observations were $\sim 260 \mathrm{eV}$ and $\sim 150 \mathrm{eV}$ respectively. It is reasonable to suppose that the broad gaussian is caused by the Doppler broadening of an iron line and that the difference of the widths is explained as the difference of the Alfven radius between two observations. As for the spectra among three phases, persistent, dip and burst, the photon indices are slightly different at the first observation ; the photon index in the persistent is larger (steeper) than that in the burst but smaller (flatter) than that found in the dip. However, at the second observation, no clear difference among spectra during three phases was found. We also investigated the spectra separately accumulated according to the sinusoidal pulse phase during persistent phase. The photon index in the upper phase of the sinusoidal curve is smaller than that in the lower phase at both observations. Because the X-ray pulsation would be due to the appearing and hiding of the part of the polar caps to our direction, the emission from the polar cap region has harder spectrum than that from the other region.

\section{References}

Fishman G.J. et al. (1995), IAUC, 6272,

Kouveiotou C.et al.(1996), NATURE, 379 , 